\title{
An integrative approach to identifying cancer chemoresistance-associated pathways
}

\author{
Shih-Yi Chao ${ }^{1}$, Jung-Hsien Chiang ${ }^{2 *}$, A-Mei Huang ${ }^{3 *}$, Woan-Shan Chang ${ }^{2}$
}

\begin{abstract}
Background: Resistance to chemotherapy severely limits the effectiveness of chemotherapy drugs in treating cancer. Still, the mechanisms and critical pathways that contribute to chemotherapy resistance are relatively unknown. This study elucidates the chemoresistance-associated pathways retrieved from the integrated biological interaction networks and identifies signature genes relevant for chemotherapy resistance.

Methods: An integrated network was constructed by collecting multiple metabolic interactions from public databases and the k-shortest path algorithm was implemented to identify chemoresistant related pathways. The identified pathways were then scored using differential expression values from microarray data in chemosensitive and chemoresistant ovarian and lung cancers. Finally, another pathway database, Reactome, was used to evaluate the significance of genes within each filtered pathway based on topological characteristics.

Results: By this method, we discovered pathways specific to chemoresistance. Many of these pathways were consistent with or supported by known involvement in chemotherapy. Experimental results also indicated that integration of pathway structure information with gene differential expression analysis can identify dissimilar modes of gene reactions between chemosensitivity and chemoresistance. Several identified pathways can increase the development of chemotherapeutic resistance and the predicted signature genes are involved in drug resistant during chemotherapy. In particular, we observed that some genes were key factors for joining two or more metabolic pathways and passing down signals, which may be potential key targets for treatment.

Conclusions: This study is expected to identify targets for chemoresistant issues and highlights the interconnectivity of chemoresistant mechanisms. The experimental results not only offer insights into the mode of biological action of drug resistance but also provide information on potential key targets (new biological hypothesis) for further drug-development efforts.
\end{abstract}

\section{Background}

The development of chemotherapy resistance is of tremendous significance to patients, researchers, and care providers who rely on conventional cytotoxic agents for the treatment of cancer. Still, the mechanisms and related biological pathways that contribute to chemotherapy resistance are relatively poorly understood. Numerous attempts have been made to mitigate or eliminate chemotherapy resistance, based-on certain assumptions about the various mechanisms, but low response rates

\footnotetext{
* Correspondence: jchiang@mail.ncku.edu.tw; amhuang@kmu.edu.tw ${ }^{2}$ Department of Computer Science and Information Engineering, National Cheng Kung University, No. 1, University Road, Tainan City 701, Taiwan ${ }^{3}$ Department of Biochemistry, Kaoshiung Medical University, Shih-Chuan 1st Road, Kaohsiung, 807, Taiwan

Full list of author information is available at the end of the article
}

and poor clinical outcomes for patients can be attributed to our inability to identify and subsequently target major molecular interactions associated with such resistance. Many genes have recently been reported to determine sensitivity to multiple drugs include drug transporters and metabolizing enzymes [1-4], and certain genes have also been demonstrated to determine sensitivity to specific chemotherapy drugs [5-7]. Other studies have attempted to estimate the chemosensitivity of cancers using genome-wide expression profile analyses, such as cDNA microarray and single nucleotide polymorphisms [8-10]. Although these studies have described genes as being capable of determining the sensitivity to chemotherapy drugs, the interactions between such genes have not been addressed, and considerable attention has focused on identifying molecular interactions associated

\section{Biomed Central}


with chemotherapy resistance. Cabusora et al. reported particular response sub-networks in the $M$. tuberculosis network after treatment with unspecific stress-inducers and comparison with antibacterial drugs [11]. To identify rational targets for combination therapy, Riedel et al. attempted to identify the biological networks implicated by differential gene expression between sensitive and resistant cell lines [12].

However these studies did not take into account the drug active pathways, including the regulatory interactivities of genes influenced by the drug. The drug active pathway plays an important role in the drug responses of the cellular system affected by the drug and the prediction of side-effects, which is also a very important issue for identifying and validating drug target genes through their regulatory relationships. Moreover, considerations should be taken of drug resistance mechanisms, including reduced intracellular drug accumulation, increased detoxification of the drug by thiol-containing molecules, increased DNA damage repair, and altered cell signaling pathways and apoptosis mediators [13]. In addition, chemotherapy drugs can be categorized based on their function, chemical structure and interaction with other drugs. Cisplatin and carboplatin, classified as DNA alkylating agents, are platinum-based chemotherapy drugs used to treat various cancers, including sarcomas, small cell lung cancer, ovarian cancer, lymphomas and germ cell tumors. These platinum-based chemotherapy drugs react with DNA in vivo by binding to and causing cross-linking of DNA which ultimately triggers apoptosis [14]. For example, cisplatin forms highly reactive, charged, platinum complexes which bind to nucleophilic groups (such as GC-rich sites) in DNA, inducing intra-strand and inter-strand DNA cross-links, as well as DNAprotein cross-links. These cross-links result in apoptosis and cell growth inhibition. When cells become resistant to cisplatin, the doses must be increased, and a large dose escalation can lead to severe multi-organ toxicities and intractable vomiting. The mechanisms of cisplatin drug resistance may include decreased intracellular accumulation of cisplatin and increased DNA repair, which also are drug resistance related pathways considered in this approach. Hence, a large biological interaction network was re-constructed by collecting from public databases DNA damage-related pathways, cell signalling-related pathways and the regulatory relationships between genes.

Combining pathway structure information mined from the re-constructed large biological interaction network with gene differential expression values, this study elucidates the particular platinum-based chemoresistance-associated pathways. Genes deemed relevant for chemotherapy resistance were also determined. Results of this study demonstrated that the identified pathways can increase chemotherapy resistance. This approach can identify pathways with a response dissimilar to that of known modes of biological action, and these new hypotheses can be used early in the drug development process to avert repeated and costly clinical trails. The major contributions of this approach are: (1) to reveal the phenomenon of chemoresistant mechanisms and related interactions between genes by combining pathway structure information with gene differential expressions; (2) to provide crossing validation candidate signature gene sets by calculating the values of betweenness centrality and degree in large complex networks; and (3) to propose new hypotheses for chemoresistant mechanisms through systems biology.

\section{Methods}

\section{Materials and databases}

This section covers the graph-theoretical properties, biological network constructions, and data sets.

\section{Graphs and networks}

Basic graph-theoretical properties and representations used by this study are as follow:

DEFINITION. A graph $G=(V, E)=(V(G), E(G))$ consists of a vertex set $V(G)$ with vertices (or nodes) $v_{i}$ $\in V(G)$, and an edge set $E(G)$ with $\left(v_{i}, v_{j}\right) \in E(G)$.

A graph $G$ with biological information yields a biological network $N_{B}$ as follows:

DEFINITION. Let $N_{B}=(V, E, \delta)$ be a network with vertices $v \in V$, edges $e \in E$, and a function $\delta: Y \rightarrow P(Y=$ $V \cup E)$ that maps vertices and edges onto their respective properties $p \in P$.

Depending on the particular network representation, in a biological network vertex properties can include genes, proteins or chemical elements, and edge properties may refer to specific interactions, such as binding or regulating. The mapping $\delta: Y \rightarrow P$ is at least subjective because for all $p \in P$, there exists a $y \in Y$ with $\delta(y)=p$.

\section{Heterogeneous biological network integration and re-} construction

To integrate heterogeneous biological networks, we identified three types of interactions relevant to a network: (i) protein interactions, such as protein-DNA binding or multi-state protein phosphorylation by kinases during signaling, (ii) regulatory reactions including co-expressions in regulons, and positive and negative regulation, and (iii) metabolic reactions. For protein interaction data, we parsed the Pathway Interaction Database (PID) [15], a highly-structured, curated collection of information about known biomolecular interactions and key cellular processes assembled into signaling pathways. Furthermore, the TRANSFAC [16] database provided information on regulatory reactions including co-expressions in regulons, and positive and negative 
regulation. For metabolic reaction data, we used the Kyoto Encyclopedia of Genes and Genomes (KEGG) $[17,18]$ to construct molecular interaction and reaction networks for metabolism. KEGG contains reaction networks of cellular processes, human diseases and drug development. Given this study's focus on identifying differential expression pathways during platinum-based chemotherapy drugs resistance, we determined diversified pathways correlated with cancer diseases, DNA repair, and metabolism for parsing and integration. Pathway selection criteria and the overall pathway sets collected in this study are listed in Additional file 1.

Our goal was to use protein interactions and regulatory reactions assembled into metabolic pathways without introducing duplicated links and elements. To merge interactions from various sources, the genes' alias names must be arranged in advance. Furthermore, we recorded the directions of interactions between genes (or proteins) as well to the graph. We joined the proteins as vertices to the integrated large network and connected them to any co-regulated genes by adding new edges. From a biological viewpoint of transcriptional relationships, a number of genes may regulate themselves or regulate each other, resulting in cyclic relationships while re-constructing the large network, which makes it more difficult to determine simple shortest paths. We dealt with this problem by merging vertices as demonstrated in Figure 1. Taking Figure 1(b) as an example, the transcription factors AR (androgen receptor) and DDIT3 (DNA-damage-inducible transcript 3) regulate their target genes and regulate each other as well. To preserve the biological truth and avoid loops being represented in the graph, vertices AR and DDIT3 were merged during the shortest paths algorithm. Next, while scoring the identified pathways according to gene expression data, each vertex (or gene) was considered separately and identically.

\section{Microarray data}

Peters et al. presented the results of a preliminary investigation into the molecular phenotype of patient-derived ovarian tumor cells in the context of sensitivity or resistance to carboplatin [19]. They correlated chemoresponse data with gene expression patterns at the level of transcription. Primary cultures of cells derived from ovarian carcinomas of individual patients $(n=6)$ were characterized using the ChemoFx assay and classified as either carboplatin sensitive $(n=3)$ or resistant $(n=3)$. Three representative cultures of cells from each individual tumor were then subjected to Affymetrix gene chip analysis $(\mathrm{n}=18)$ using U95A human gene chip arrays. They identified numbers of differentially expressed genes that define transcriptional differences between chemosensitive and chemoresistant cells and temporal responses to carboplatin expressed in an ex vivo setting.
Gabriela et al. investigated the response to cisplatin of a panel of NSCLC cell lines and found an inverse correlation between sensitivity and damage formation resulting from this agent [20]. Further analysis of multiple alternate cellular end-points including cell cycle analysis, apoptosis and gene expression changes, revealed cisplatin damage tolerance to be a mechanism of chemoresistance in this model system. Both gene expression data sets were available through the Gene Expression Omnibus (GEO) at NCBI [21] (GEO platform accession number GDS 1381 and GSE 6410, respectively).

\section{Systems and implementation System overview}

A system flow diagram of the corresponding processes is shown in Figure 2. The system is composed of four major parts, including heterogeneous biological network integration, the selection of seed nodes, identification of pathways, and analysis of differential expressions. As described in the previous section, the large integrated biological network was constructed and stored in MySQL database. By stripping away unambiguous vertices according to the genes' official symbols and the duplicated interactions between them, the $\mathrm{k}$-shortest path algorithm could be implemented to obtain the shortest pathways for given seed nodes. The seed nodes are particular nodes given by users or selected from transcription factors, and paths between them are identified by the k-shortest path algorithm. The identified pathways were scored using gene expression values as metrics for weighted edges. Finally, the top scoring $n$ pathways were selected and further analyzed.

\section{Pathway identification}

The first step in the pathway identification process was seed node selection. Here, particular vertices were tagged as "seed nodes", and the shortest paths between them were identified by Yen's algorithm [22]. From a mathematical viewpoint, this procedure extracts from the large, integrated biological network a pathway that is spanned by selected seed nodes. Yen's algorithm is still the best known approach to the k-shortest simple paths problem with respect to its worst case running time, i.e., $\mathrm{O}(k n(m+n \log n))$ time for a graph with $\mathrm{m}$ vertices and $n$ edges. Seed nodes were determined either by users' interesting genes [23] or were selected by biologists in advance. The criteria of selecting seed nodes are listed as follows: (i) genes with functional annotations such as 'DNA damage', 'DNA repair' and other related functional annotations [24], (ii) genes that are known transcription factors and are implicated in drug resistance [25], and (iii) genes that have been reported to have significantly altered expression patterns between platinum-based drugs chemosensitive and chemoresistant cells. We were specifically interested in a 


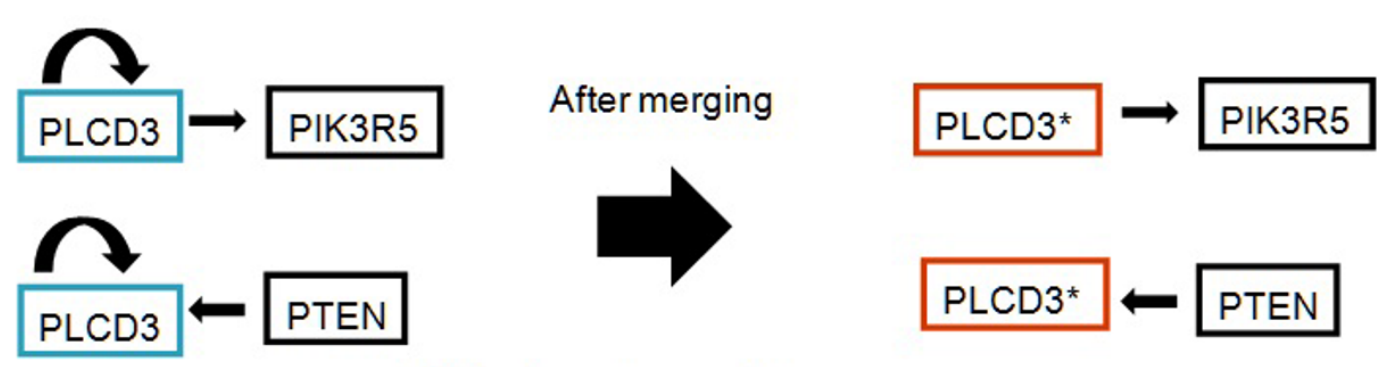

(a) Merging auto-regulate genes
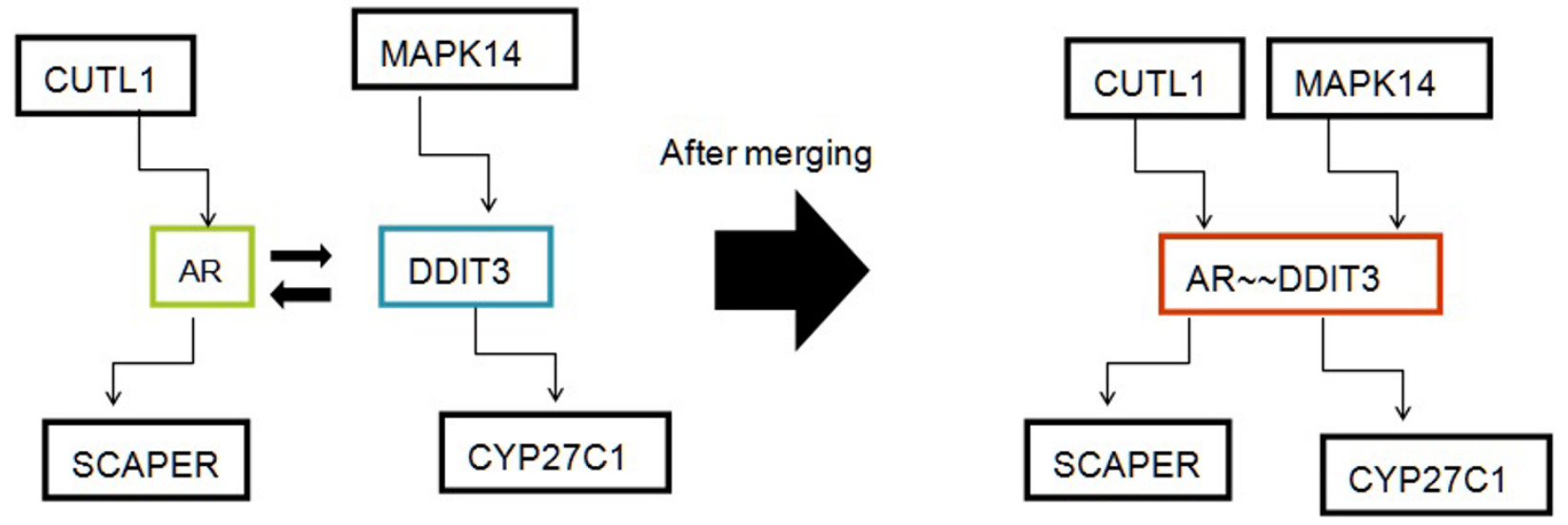

(b) Merging genes that regulate each other

Figure 1 An overview of merging vertices. Figure 1(a) shows the concept of merging auto-regulate genes. From a biological viewpoint of transcriptional relationships, a number of genes may regulate themselves or regulate each other, resulting in cyclic relationships while reconstructing the large network, which makes it more difficult to determine simple shortest paths. Figure 1(b) demonstrates how to merge genes that regulate each other. Transcription factors, AR (androgen receptor) and DDIT3 (DNA-damage-inducible transcript 3), regulate their target genes and regulate each other as well. To preserve the biological truth and avoid loops being represented in the graph, vertices AR and DDIT3 were merged during the shortest paths algorithm. Next, while scoring the identified pathways according to gene expression data, each vertex (or gene) was considered separately and identically.

transcription factor CEBPD (CCAAT/enhancer binding protein (C/EBP), delta) which has been implicated in tumor suppression [26-29]. Interestingly, CEBPD exhibits a pro-oncogenic function in cisplatin resistance phenotype [23]. Therefore, we conducted a gene expression study to further identify CEPBD-regulated genes which might contribute to cisplatin resistance (Huang, el al. unpublished data). The merged CEBPD-regulated genes are listed as user-interested genes in Table 1.

\section{Scoring and filtering pathways}

The main procedure of pathway scoring was calculating the differential expression values for the genes as metrics for weighted edges in the pathway. In this study, genes, proteins and other cellular components were coded as vertices which are connected by their edges to represent the interactions in the integrated biological network. However, the scoring step assumes weights on the edges for summing scores, and such edge weights must be calculated from the vertices' scores. Therefore, the identified pathway was subsequently transformed and represented as a line graph in which the edges represent genes, proteins and other cellular components, and vertices refer to interactions. Edges can then be directly weighted by gene expression values.

REMARK. Give a biological network $N_{B}$, its line graph $L\left(N_{B}\right)$ is a graph such that each vertex of $L\left(N_{B}\right)$ represents an edge of $N_{B}$; and two vertices of $L\left(N_{B}\right)$ are adjacent if and only if their corresponding edges share a common endpoint in $N_{B}$.

To filter and identify the "significant pathways" (e.g., connected sets of genes with high levels of differential expression) we followed Ideker et al.'s statistical scoring system which captures the amount of gene expression change in a given pathway [30]. To rate the biological activity in a particular pathway, we first assessed the significance of the differential expression for each gene. 


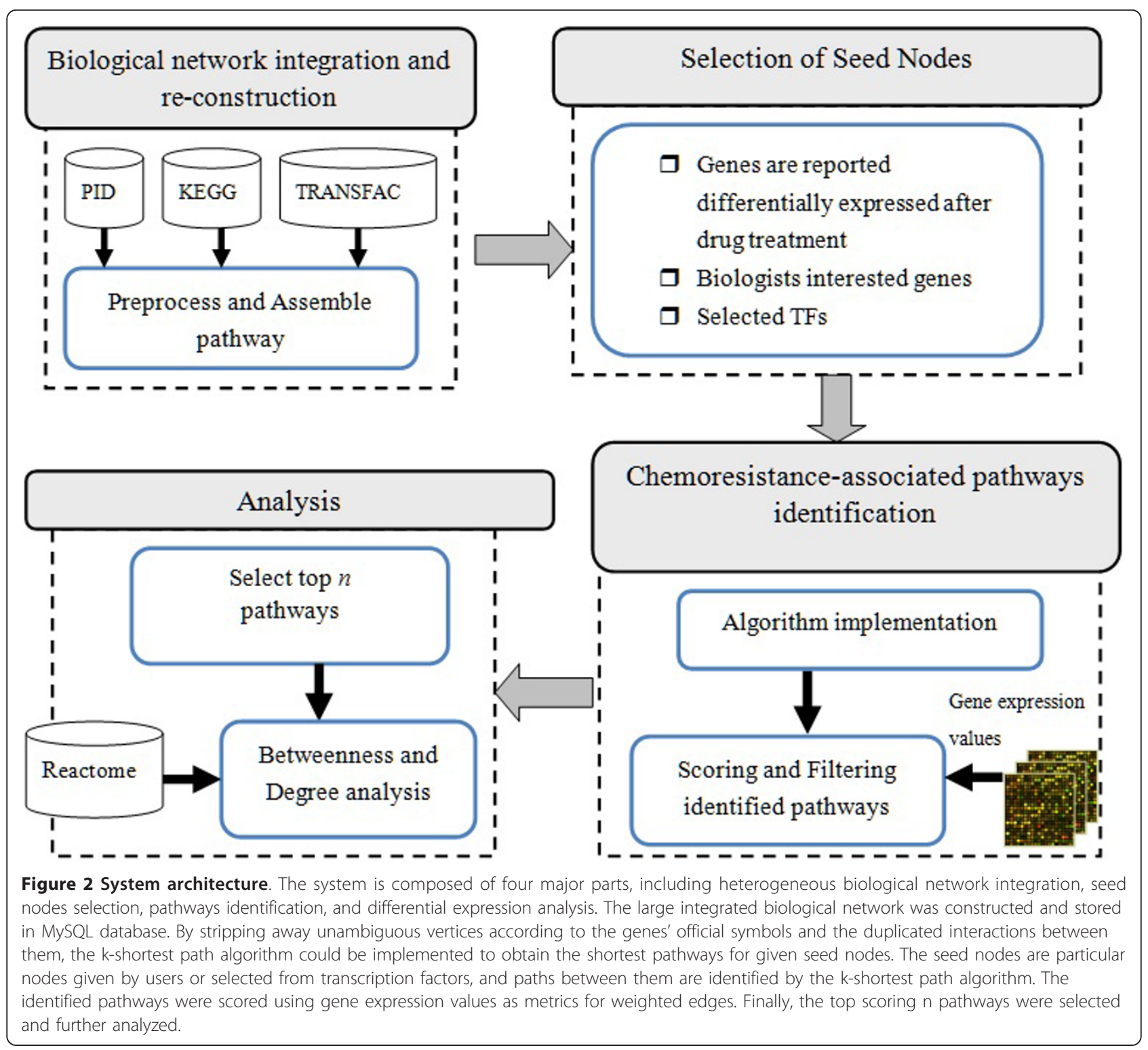

We extracted the $p$-value $p_{m}$ for each expressed gene $m$ in the microarray data and then converted the $p_{m}$ into a $z$-score by Formula 1.

$$
z_{m}=\Phi^{-1}\left(1-p_{m}\right)
$$

where $\Phi^{-1}$ denotes the inverse normal cumulative distribution function. In random data, $p$-values are distributed uniformly from 0 to 1 and $z$-scores follow a standard normal distribution, with smaller $p$-values corresponding to larger $z$-scores. The aggregate score of a set of genes in a pathway can be calculated by summing the $z_{m}$ over all $m$ in the pathway,

$$
z_{N_{B}}=\frac{1}{\sqrt{m}} \sum_{m \in N_{B}} z_{m}
$$

Under this scoring function, the pathways of all sizes can be compared, with a high score indicating a biologically active pathway and pathways were then filtered by an assigned threshold score. In summary, the $k$-shortest path approach guarantees effective pathway identification through a particular set of seed nodes. The scoring functions (Formulas 1 and 2) contribute an appropriate constraint filtering pathways. Once the top $n$ pathways have been selected, the analysis of pathways process can be performed.

\section{Analyze the pathway signatures}

The main purpose of performing pathway intersections is to determine whether different cancers have identical chemoresistant mechanisms. Comparing two pathways requires the identification of the corresponding vertices. The correspondences between vertices in the pathways 


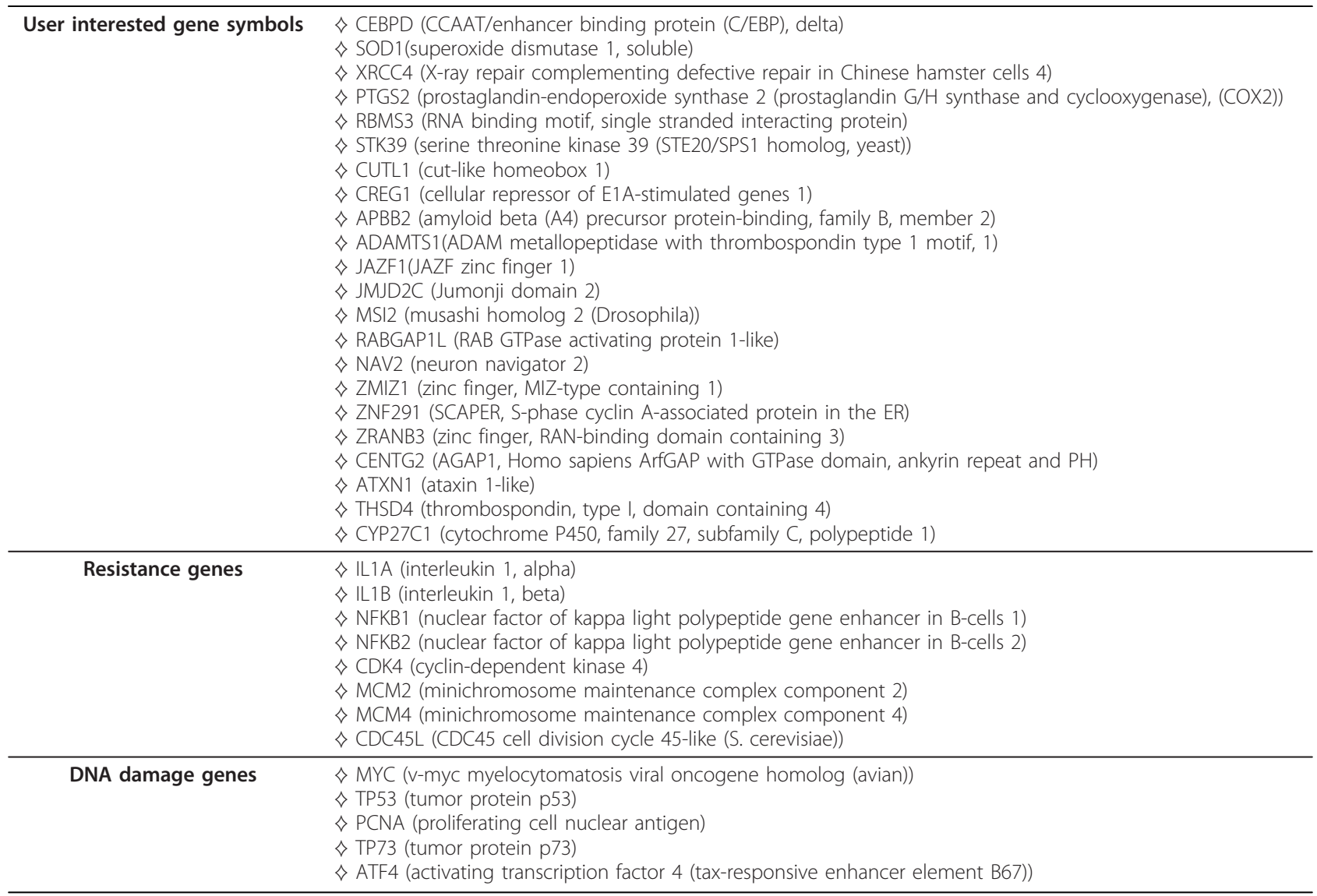

Seed nodes were determined either by users' interesting genes [23] or were selected by biologists in advance. The criteria of selecting seed nodes are listed as follows: (i) genes with functional annotations such as 'DNA damage', 'DNA repair' [24], (ii) genes that are known transcription factors and are implicated in drug resistance [25], and (iii) genes that have been reported to have significantly altered expression patterns between platinum-based drugs chemosensitive and chemoresistant cells.

are given by matching the genes' official symbols. In general, the correspondences can be many-to-many for the reason that a vertex (e.g. representation of an enzyme) may catalyze different reactions in the pathway and may be catalyzed by multiple vertices as well. In other words, graph comparison is an NP-hard problem which typically can only be addressed by exhaustive enumeration techniques. Here, we present an approach using the vertices and edges labels of the given pathways. Consider two graphs, $N^{1}{ }_{\mathrm{B}}\left(V_{1}, E_{1}\right)$ and $N^{2}{ }_{\mathrm{B}}\left(V_{2}\right.$, $\left.E_{2}\right)$, and a matrix representing the correspondences between $V_{1}$ and $V_{2}$. Let $s(e)$ and $t(e)$ be the origin and terminal nodes of edge $e$ so the intersection of the pathways is defined as:

$$
\Omega\left(N^{1}{ }_{B}, N^{2}{ }_{B}\right)=\left\{\begin{array}{c}
v, e: \delta\left(v_{1}\right)=\delta\left(v_{2}\right) \wedge\left[\delta\left(e_{1}\right)=\delta\left(e_{2}\right)\right. \\
\text { if } \left.\delta\left(s\left(e_{1}\right)\right)=\delta\left(s\left(e_{2}\right)\right) \wedge \delta\left(t\left(e_{1}\right)\right)=\delta\left(t\left(e_{2}\right)\right)\right]
\end{array}\right\}
$$

where $v_{1} \in V_{1}, v_{2} \in V_{2}$ and $e_{1} \in E_{1}, e_{2} \in E_{2}$. In other words, the intersection between all $v_{1} \in V_{1}$ and $v_{2} \in V_{2}$, and the intersection between corresponding edges $e_{1} \in E_{1}, e_{2} \in E_{2}$ under the condition that $\forall v_{1}, v_{2}: \delta\left(v_{1}\right)=\delta\left(v_{2}\right)$ and $\forall e_{1}, e_{2}: \delta\left(e_{1}\right)=$ $\delta\left(e_{2}\right)$. An edge $e \in N_{\mathrm{B}}^{1}$ is selected if both the originating and terminating vertices have $\delta$-corresponding vertices in $N_{\mathrm{B}}^{2}$.

To assess the importance of genes within each filtered pathway, we also implemented the betweenness centrality and degree centrality for each node. The degree and betweenness centrality of genes were calculated using the Reactome database [31] as a base to cross validate our experimental results. The betweenness centrality of a node in a network topology measures how many shortest paths go through that node. If $b_{i}$ is the ratio of the number of shortest paths between a pair of nodes in the network that pass through node $i$ and the total number of shortest paths between those two nodes, the unscaled betweenness of node $i$ is $B_{i}^{\prime}=\sum_{\text {all pairs }} b_{i}$, and the (scaled) betweenness centrality is

$$
B_{i}=\frac{2 B_{i}^{\prime}}{(n-1)(n-2)}
$$

where $n$ is the number of nodes in the network. The betweenness centrality is positive and always less than 
or equal to 1 for any network. The degree of a node in a network is the number of connections or edges by which the node is related with other nodes. Degree centrality is the number of links that connect the node to the network divided by the number of nodes in the network minus 1 . It is a local measure that does not account for network context. However, changes in nodes with high degree centrality are likely to influence a large number of nodes in the network. The degree centrality was calculated by Formula (5).

$$
D(v)=\frac{\operatorname{deg}(v)}{n-1}
$$

Formula (5) indicates the degree centrality of an undirected graph. As for a vertex representing the gene (or protein) in an undirected graph, the higher the degree, the more reactions it interacts with and the more important the vertex is.

\section{Results and Discussion}

As described in previous section, we integrated the PID, KEGG and TRANSFAC public databases, and further eliminated duplicated reactions and elements. Accordingly, 8173 genes and 9308 interactions were retained, for which both detailed and summarized database results are presented in Table 2. In the next section, we present the experimental results and analysis of the pathway intersections.

\section{Significant pathways in ovarian cancer}

Ovarian cancer is among the most malignant of all lethal diseases in women. Currently, the preferred treatment regimen for ovarian cancer is combination chemotherapy primarily with platinum-based drug such as cisplatin or carboplatin. While this treatment course has shown promising effects in a high percentage of cases, the development of chemoresistance is a significant hurdle to successful treatment outcomes [32]. Hence, we have focused our research on elucidating the mechanisms induced by chemotherapeutic agents; that is, the DNA damage, DNA repair, and apoptosis in ovarian cancer cells resulting from platinum-based drug chemotherapy and chemoresistance. One of the significant pathways identified from the ovarian cancer expression data is shown in Figure 3, with the notations presented in Additional file 2.

As shown in Figure 3, c-KIT (also called KIT or C-kit receptor) is one of target genes regulated by CEBPD, a growth factor receptor exhibiting tyrosine kinase activity. Moreover, c-KIT is not only a biochemical marker; its involvement in autocrine, paracrine or endocrine growth loops may represent a molecular mechanism behind aggressive tumor growth [33,34]. Raspollini et al. performed an immunohistochemistry analysis of 56 patients with advanced serous ovarian carcinomas using archival paraffin-embedded specimens and demonstrated that c-KIT was expressed in ovarian carcinoma and was statistically correlated with chemotherapy resistance [35]. C-KIT expression has been shown to be statistically correlated with the progression of disease after first-line chemotherapy. Moreover, c-KIT was identified by our pathway mining procedure with $\mathrm{p}$-value $<0.05$ (listed in Table 3) by t-test calculated from the ovarian expression data, indicating this approach identify genes involved in chemoresistant mechanisms.

Table 2 Lists of \# of genes and relations in integrated database

\begin{tabular}{|c|c|c|c|}
\hline \multicolumn{4}{|c|}{ Statistics information of integrated databases } \\
\hline Database & Organism & \# of gene & \# of relation \\
\hline PID+KEGG+TRANSFAC & Homo sapiens & 8173 & 9308 \\
\hline Reactome & Homo sapiens & 538 & 31240 \\
\hline \multicolumn{4}{|c|}{$\begin{array}{l}\text { Statistics information on each of the three } \\
\text { databases }\end{array}$} \\
\hline Database & \# of TFs & \# of target gene parsed & $\begin{array}{l}\text { \# of pairing regulate relation } \\
\text { parsed }\end{array}$ \\
\hline TRANSFAC & 157 & 825 & 529625 \\
\hline Database & $\begin{array}{c}\text { \# of } \\
\text { pathways }\end{array}$ & $\begin{array}{c}\text { \# of gene, protein, enzyme } \\
\text { parsed }\end{array}$ & \# of relation parsed \\
\hline$P I D+K E G G$ & 197 & 18937 & 8880 \\
\hline PID & 60 & & \\
\hline KEGG & 137 & & \\
\hline
\end{tabular}

We integrated the PID (the date of version, July 15, 2008), KEGG (release 47.0, July 1, 2008) and TRANSFAC public databases (version 7.0), and further eliminated duplicated reactions and elements. Accordingly, 8173 genes and 9308 interactions were remained. To assess the importance of genes within each filtered pathway, we also implemented the betweenness centrality and degree centrality for each node. The degree and betweenness centrality of genes were calculated using the Reactome database [31] as a base to cross validate our experimental results. Pathways downloaded from PID and KEGG were parsed by batch processing. A gene (or protein) may be involved in several pathways, which means some genes were repeated. Therefore, the number of parsed entity (including genes, proteins, and enzymes) was 18937 . Moreover, one gene may be regulated by several TFs, or one TF may regulate numerous target genes. As a result, the total number of pairing regulate relation parsed was 529625. 


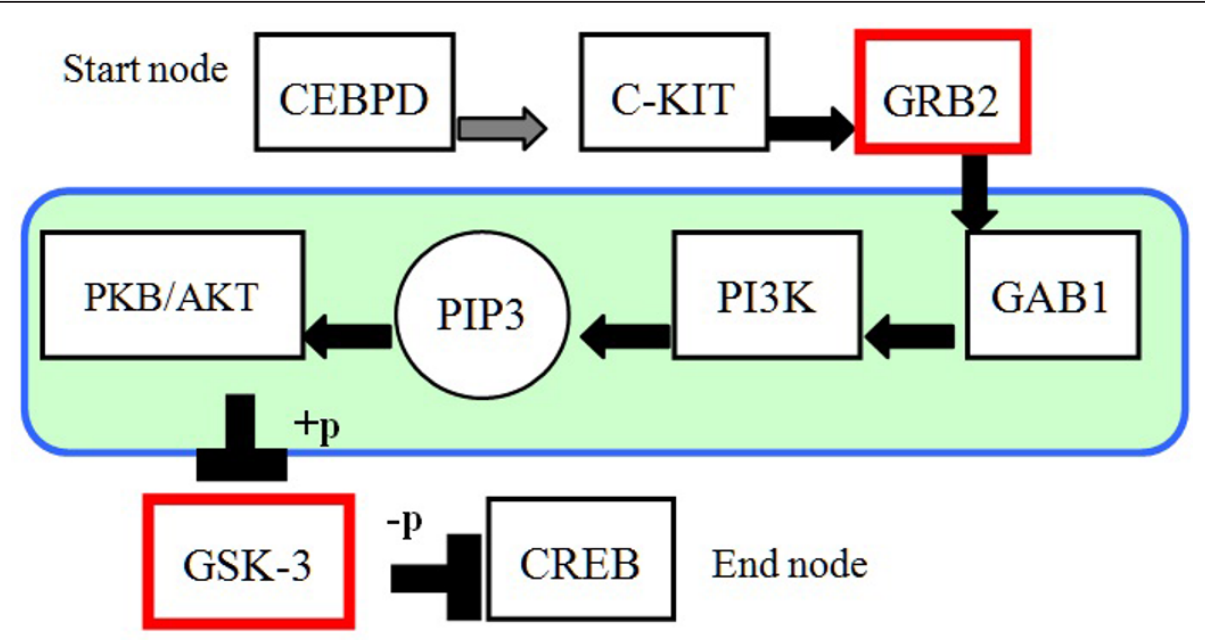

Figure 3 One experimental result of identified pathways from ovarian expression data. This diagram shows one of identified signature pathways. Genes represented by red squares indicate the connected nodes; that is, these genes connect pathways. Connected nodes are key factors for joining two or more metabolic pathways or passing down signals. The blue square indicates the PI3K/AKT pathway. The PI3K/AKT cascade plays an important role in the resistance of ovarian cancer cells to cisplatin in vitro. This pathway is therefore an attractive target for the development of novel anticancer agents.

As indicated in Figure 3, the PI3K (Phosphatidylinositol 3-kinas)/AKT gene family are involved as well. The PI3K pathway is stimulated as a physiological consequence of many growth factors and regulators. In addition, the activation of the PI3K pathway results in disturbances of cell growth and survival control, which contributes to a competitive growth advantage, metastatic competence and, frequently, therapy resistance [36]. Therefore, this pathway is an attractive target for the development of novel anticancer agents. The PI3K/ Akt cascade plays an important role in the resistance of ovarian cancer cells to cisplatin in vitro. Ohta et al. investigated whether the inhibition of PI3K increased the efficacy of cisplatin in an in vivo ovarian cancer model [37]. Blocking the PI3K/Akt cascade with a PI3K inhibitor (wortmannin) increased the efficacy of cisplatin-induced inhibition of intra-abdominal dissemination and production of ascites in athymic nude mice inoculated ip with the Caov-3 human ovarian cancer cell line. In addition, wortmannin increased the efficacy of cisplatin-induced apoptosis in tumors cells. Ohta et al. also confirmed that wortmannin blocked Akt phosphorylation and the downstream targets of the PI3K/Akt cascade, such as BAD (Bcl-2-associated death protein) and nuclear factor ${ }_{-\mathrm{k}} \mathrm{B}$ in vivo by immunohistochemical staining and Western blotting. Moreover, Lee et al. used human ovarian cancer cell OVCAR-3 and cisplatin-resistant subclone OVCAR-3/CDDP cells to study the roles of PIK3CA (alias name PI3K) and PTEN on the resistance of human ovarian cancer cells to cisplatin-induced apoptosis [38]. They systematically examined the expressions of apoptosis regulating proteins

Table 3 Genes identified in figure 3 with p-value $<0.05$ by t-test

\begin{tabular}{|c|c|c|c|c|}
\hline Gene Symbol & $\begin{array}{l}\text { Ovarian } \\
p \text {-value }\end{array}$ & $\begin{array}{c}\text { Betweenness } \\
\text { (mean = } \\
3.8 \mathrm{E}-4)\end{array}$ & $\begin{array}{c}\text { Degree } \\
\text { (mean = } \\
9.71 \mathrm{E}-4)\end{array}$ & $\begin{array}{l}\text { Connected } \\
\text { nodes }\end{array}$ \\
\hline $\begin{array}{c}\text { c-KIT } \\
\text { (v-kit Hardy-Zuckerman } 4 \text { feline sarcoma viral oncogene homolog, also called KIT or } \\
\text { C-kit receptor) }\end{array}$ & $3.53 \mathrm{E}-07$ & 0.00178 & 0.006483 & \\
\hline $\begin{array}{l}\text { GRB2 } \\
\text { (growth factor receptor-bound protein 2) }\end{array}$ & 5.74E-06 & 0.020155 & 0.023064 & V \\
\hline $\begin{array}{c}\text { AKT2 } \\
\text { (v-akt murine thymoma viral oncogene homolog 2) }\end{array}$ & 0.022784 & $3.89 \mathrm{E}-04$ & 0.002369 & V \\
\hline $\begin{array}{c}\text { PIK3CG } \\
\text { (phosphoinositide-3-kinase, catalytic, gamma polypeptide) }\end{array}$ & $1.29 \mathrm{E}-04$ & $9.77 \mathrm{E}-05$ & 0.001247 & \\
\hline
\end{tabular}

Genes listed in this table are significant in ovarian expression data with $p$-value $<0.05$. Moreover, the betweenness and degree centrality of each gene are also listed. Both GRB2 and AKT2 gene are connected nodes; which indicates they pass down signals between pathways. GRB2 with significant betweenness and degree centrality indicates it has potential to act as a "hub node" in biological interaction networks and to involve in chemoresistant mechanisms as well. 
and PI3K/Akt signaling proteins, finding that OVCAR$3 / \mathrm{CDDP}$ cells were 4.8 -fold more resistant to cisplatin than OVCAR-3 cells following $72 \mathrm{~h}$ exposure to the drug. This resistance correlated with reduced susceptibility to cisplatin-induced apoptosis. Apoptotic proteins were differentially expressed in the OVCAR-3/CDDP cells, resulting in the inhibition of Bax translocalization. Their experimental results indicate that the development of resistance in OVCAR-3 cells is derived from increasing PIK3CA transcription and reducing of PTEN expression. These alterations confer resistance to cisplatin through the activation of PI3K. These in vivo results support the proposition that our algorithm can identify chemoresistance-associated pathways.

In Figure 3, genes are represented by red squares indicating the connected nodes; that is, these genes connect two pathways. Connected nodes are key factors for joining two or more metabolic pathways or passing down signals. Taking GRB2 (Growth factor receptor-bound protein 2) as an example, L'Esperance et al. [39] found that upregulated genes in post chemotherapy ovarian tumors included a substantial number of genes with previously implicated in mechanisms of chemoresistance including COX2 and tumorigenesis, GRB2. As seen in Figure 3, AKT was also identified as a connected gene, and had significant betweenness centrality and degree values (shown in Table 3), indicating that AKT has potential to act as a "hub node" in biological interaction networks and be involved in chemoresistant mechanisms as well [40].

\section{Significant results following pathway intersections}

The main analysis of this experiment focused on whether different cancers identical chemoresistant mechanisms and whether these chemoresistant mechanisms share some genes in common. After performing intersection by Formula (3), 88 pathways remained (the Additional file 3). The following sections include further analysis.

The major goals of this analysis were: (1) to explore pathways or genes involved in chemoresistant mechanisms; (2) to delineate how these genes or pathways interact with each other; (3) to test whether the $p$-values of the genes in this pathway are significantly differentially expressed; (4) to analyze the betweenness centrality (Formula 4 ) and degree (Formula 5) values of genes in this pathway; and (5) to identify the chemoresistanceassociated genes.

As shown in the Diagram 4, several pathways contributed to this result: the colorectal cancer related pathway, the hedgehog signaling pathway, the WNT signaling pathway and the notch signaling pathway. In addition, some other pathways, such as the p53 signaling pathway, the MAPK signaling pathway, and the focal adhesion were partially involved as well. Platinum-based cancer drugs (including cisplatin and carboplatin) are among the most potent anti-tumor agents, displaying clinical activity against a wide variety of solid tumors. Its cytotoxic mode of action is mediated by its interaction with DNA to form DNA adducts, primarily intrastrand crosslink adducts, which activate several signal transduction pathways, including those involving ATR, p53, p73, and MAPK, and culminate in the activation of apoptosis [41]. Resistance mechanisms that limit the extent of DNA damage include reduced drug uptake, increased drug inactivation, and increased DNA adduct repair. Mechanisms that inhibit the propagation of the DNA damage signal to the apoptotic machinery include loss of damage recognition, overexpression of HER-2/neu, activation of Akt (indicated by the red square in Figure 4), and loss of p53 function [5]. The molecular signature defining the resistant phenotype varies between tumors, and the number of resistance mechanisms activated in response to selection pressures dictates the overall extent of resistance. This experimental result implies the complicated nature of chemoresistance progression, which reflects that several mechanisms contribute to the multi-factorial nature of the chemoresistance problem. Although ovarian and lung cancers are assorted malignancies, based on the results of the pathway intersections experiment, several mechanisms are together responsible for platinum-based chemoresistance.

Table 4 shows the genes that involved in intersected pathways with $p$-value $<0.05$ calculated in the expression data for ovarian cancer and lung cancer. For example, the expression values for the AKT gene, are not only significantly different in both cancer expression data sets, but the value of betweenness centrality and degree are higher than $3.8 \mathrm{E}-4$ and $9.71 \mathrm{E}-4$ (the respective average values). In biological terms, the betweenness centrality of a gene measures how many pathways or signal transductions go through that gene. Our experimental result indicates that the AKT gene plays an important role in chemoresistance-associated pathways. Gagnon et al. suggested that some Akt isoforms, such as Akt2 and Akt3, are involved in chemoresistance to cisplatin and that these isoforms could be putative targets for gene therapy for uterine cancers [42]. They performed biological experiments to demonstrate that Akt activity was directly involved in chemoresistance to cisplatin and to find Akt phosphorylation in KLE cells since it was a wild-type expressing PTEN cancer cell line. As shown in Table 4, PTEN was the first tumor suppressor gene to be identified in the phosphatase family, and the principal function of its gene product appears to be dephosphorylation of the second messenger PIP3 [43]. The expression of PTEN in two independent glioblastoma cell lines results in the disruption of 


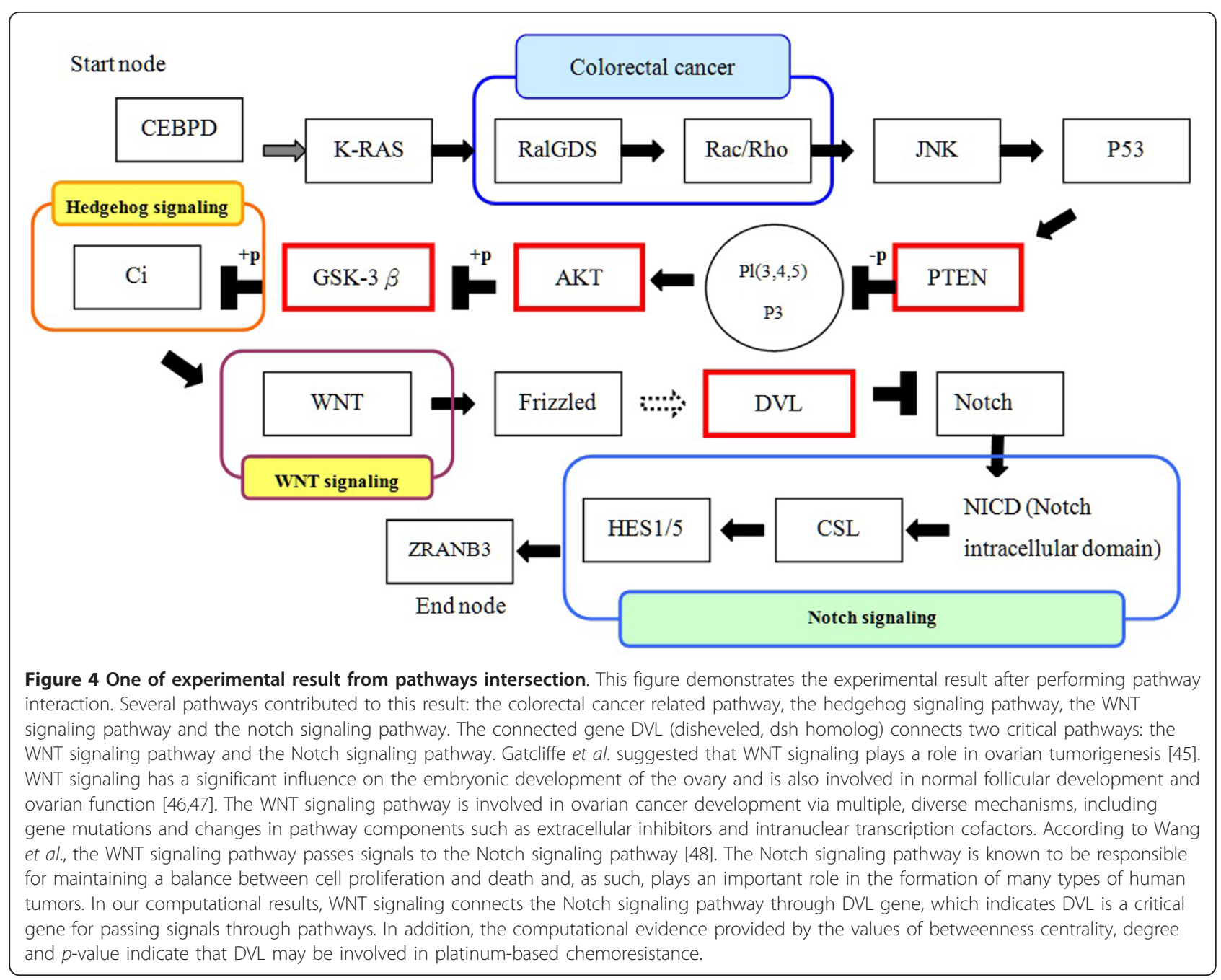

Table 4 Genes identified in figure 4 with $p$-value $<0.05$ by t-test

\begin{tabular}{|c|c|c|c|c|c|}
\hline Gene Symbol & $\begin{array}{l}\text { ovarian } \\
p \text {-value }\end{array}$ & $\underset{p \text {-value }}{\text { lung }}$ & $\begin{array}{l}\text { Betweenness } \\
(\text { mean }=3.8 \mathrm{E}-4)\end{array}$ & $\begin{array}{c}\text { Degree } \\
(\text { mean }=9.71 \mathrm{E}-4)\end{array}$ & Connected nodes \\
\hline $\begin{array}{c}\text { KRAS } \\
\text { (v-Ki-ras2 Kirsten rat sarcoma viral oncogene homolog) }\end{array}$ & $6.23 \mathrm{E}-04$ & 0.001393 & 0.00703 & 0.009226 & \\
\hline $\begin{array}{c}\text { TP53 } \\
\text { (tumor protein p53) }\end{array}$ & 0.011083 & 1.82E-04 & 0.046039 & 0.029049 & \\
\hline $\begin{array}{c}\text { AKT } \\
\text { (v-akt murine thymoma viral oncogene homolog) }\end{array}$ & $1.87 \mathrm{E}-05$ & $4.60 \mathrm{E}-06$ & 0.009775 & 0.013091 & $\mathrm{~V}$ \\
\hline $\begin{array}{c}\text { GSK3 } \beta \\
\text { (glycogen synthase kinase } 3 \text { beta) }\end{array}$ & $3.09 \mathrm{E}-06$ & $1.81 \mathrm{E}-04$ & 0.003932 & 0.006483 & $\mathrm{~V}$ \\
\hline $\begin{array}{c}\text { WNT } \\
\text { (wingless-type MMTV integration site family) }\end{array}$ & 0.009519 & 0.002234 & $1.51 \mathrm{E}-04$ & 4.99E-04 & \\
\hline $\begin{array}{c}\text { PTEN } \\
\text { (phosphatase and tensin homolog) }\end{array}$ & 0.001494 & 0.016189 & 0.002282 & 0.002618 & $\mathrm{~V}$ \\
\hline $\begin{array}{c}\text { DVL } \\
\text { (dishevelled, dsh homolog } 1 \text { (Drosophila)) }\end{array}$ & $1.95 \mathrm{E}-08$ & $1.80 \mathrm{E}-05$ & 0.001653 & 0.002618 & $\mathrm{~V}$ \\
\hline $\begin{array}{c}\text { HES1 } \\
\text { (hairy and enhancer of split 1, (Drosophila)) }\end{array}$ & 0.005831 & $2.82 \mathrm{E}-07$ & $4.08 \mathrm{E}-04$ & 0.001745 & \\
\hline
\end{tabular}

Genes listed in table 4 are significant in both ovarian and lung expression data with p-value $<0.05$. Four connected nodes were identified. These genes also had significant betweenness and degree centrality. PTEN was the first tumor suppressor gene to be identified in the phosphatase family, and the principal function of its gene product appears to be dephosphorylation of the second messenger PIP3 [43]. The expression of PTEN in two independent glioblastoma cell lines results in the disruption of downstream signaling of PI3K to Akt and Bad [44]. Thus, when PTEN is present, Akt phosphorylation is blocked and apoptosis mechanisms may be activated. The importance of Akt and PTEN genes are as well revealed by this work, which illustrates the accuracy and efficiency of our algorithm. 
downstream signaling of PI3K to Akt and Bad [44]. Thus, when PTEN is present, Akt phosphorylation is blocked and apoptosis mechanisms may be activated. The importance of Akt and PTEN genes are as well revealed by this work, which illustrates the accuracy and efficiency of our algorithm.

As indicated in Figure 4, the connected gene DVL (disheveled, dsh homolog) connects two critical pathways: the WNT signaling pathway and the Notch signaling pathway. Gatcliffe et al. suggested that WNT signaling plays a role in ovarian tumorigenesis [45]. The WNT pathway participates in many physiologic events in embryogenesis and adult homeostasis including cell fate specification, control of proliferation, and migration. WNT signaling has a significant influence on the embryonic development of the ovary and is also involved in normal follicular development and ovarian function $[46,47]$. The WNT signaling pathway is involved in ovarian cancer development via multiple, diverse mechanisms, including gene mutations and changes in pathway components such as extracellular inhibitors and intranuclear transcription cofactors. According to Wang et al., the WNT signaling pathway passes signals to the Notch signaling pathway [48]. The Notch signaling pathway is known to be responsible for maintaining a balance between cell proliferation and death and, as such, plays an important role in the formation of many types of human tumors. In our computational results, WNT signaling connects the Notch signaling pathway through DVL gene, which indicates DVL is a critical gene for passing signals through pathways. In addition, the computational evidence provided by the values of betweenness centrality, degree and $p$-value indicate that DVL may be involved in platinumbased chemoresistance.

\section{The signature chemoresistance-associated genes}

Most of the results analyzed in the previous section are supported by known biological evidence, which indicates that this work is able to predict candidate chemoresistance-associated genes. We were particularly interested in CEPBD (CCAAT/enhancer binding protein delta) and its transcriptional regulated gene, SOD1 $(\mathrm{Cu} / \mathrm{Zn}$-superoxide dismutase). Several reports have implicated CEBPD as a suppressor gene [26-29]. According to Hour et al., the expression of the CEPBD was induced by cisplatin and specifically elevated in a cisplatin resistant subline and transactivated SOD1 gene expression in the human bladder urothelial carcinoma NTUB1 cell line [23]. This study revealed a novel role for CEBPD in conferring drug resistance. Therefore, we suspected CEBPD is involved in ovarian and lung chemoresistance as well. Moreover, as shown in Figure 5, pathways including the gene CEBPD and SOD1 were the shortest

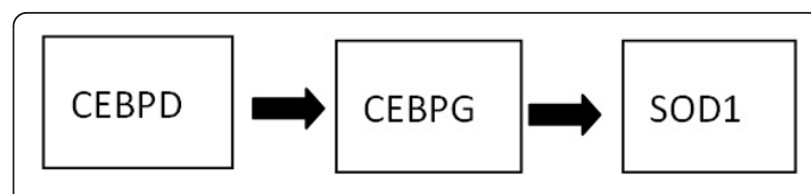

Figure 5 The shortest pathway identified by our system. Pathways including the gene CEBPD and SOD1 were the shortest pathways in our computational results, which indicates SOD1 ( $p$ value $=4.01 \mathrm{E}-04$ ) does not interact with other genes or pathways. We were curious about what caused the chemoresistant mechanism after SOD1 was regulated. Cisplatin caused DNA damage as well as reactive oxygen species (ROS), which triggered cell cycle arrest or/and apoptosis. Cisplatin induced CEBPD by an as of yet unidentified mechanism which activated the SOD1 gene expression. Superoxide anion $\left(\mathrm{O}^{\circ}\right)$ is dismutated by SOD1 and converted to $\mathrm{H}_{2} \mathrm{O}_{2}$ which can be further neutralized to water and oxygen by catalase [23]. The reduced ROS levels in their model caused the cisplatin-resistant phenotype. These results call for an assessment of CEBPD and SOD1 expression in bladder tumors as a potential means of predicting cisplatin resistance. According to our computational results, SOD1 has significant differential expressions between chemosensitive and chemoresistant array data and is activated by CEBPD as well. We may make a reasonable assumption that this phenomenon occurs in ovarian chemoresistance. Based on this biological evidence and our computational experiment results, we can infer that SOD1 plays a critical role in ovarian chemoresistance.

pathways in our computational results, which indicates SOD1 ( $p$-value $=4.01 \mathrm{E}-04)$ does not interact with other genes or pathways. We were curious about what caused the chemoresistant mechanism after SOD1 was regulated. Cisplatin caused DNA damage as well as reactive oxygen species (ROS), which triggered cell cycle arrest or/and apoptosis. Cisplatin induced CEBPD by an as of yet unidentified mechanism which activated the SOD1 gene expression. Superoxide anion $\left(\mathrm{O}^{*-}\right)$ is dismutated by SOD 1 and converted to $\mathrm{H}_{2} \mathrm{O}_{2}$ which can be further neutralized to water and oxygen by catalase [23]. The reduced ROS levels in their model caused the cisplatinresistant phenotype. These results call for an assessment of CEBPD and SOD1 expression in bladder tumors as a potential means of predicting cisplatin resistance. According to our computational results, SOD1 has significant differential expressions between chemosensitive and chemoresistant array data and is activated by CEBPD as well. Do the reduced ROS levels caused by SOD1 in ovarian chemotherapy results in the resistant phenotype as well? We may make a reasonable assumption that this phenomenon occurs in ovarian chemoresistance. Based on this biological evidence and our computational experiment results, we can infer that SOD1 plays a critical role in ovarian chemoresistance.

As shown in Figure 4, CEBPD interacts with KRAS as well and led to a domino effect that may cause chemoresistance. It was found that mutations in this candidate gene, KRAS, are one of the most frequent genetic 
abnormalities in ovarian carcinoma [49]. In other words, KRAS mutation is a common event in ovarian cancer primarily in carcinomas characterized by lower grade, lower FIGO stage, and mucinous histotype. The KRAS mutational status is not a prognostic factor for patients treated with standard therapy. However, in line with experience from colorectal cancer and NSCLC, it may prove important for predicting the response to EGFRtargeted therapies [50]. Thus far, there is no biological evidence directly indicating KRAS gene is involved in platinum-based chemoresistance but, from the computational experiment results, we may infer that KRAS plays a critical role in chemoresistance. More computational results with high scores of intersected pathways are provided in Additional file 4, and analysis of these data may reveal new chemoresistant mechanisms.

\section{Conclusions}

Although platinum-based chemotherapeutic agents are widely used for the treatment of endometrial, cervical and breast cancers, chemoresistance caused by molecular mechanisms still remains a major therapeutic problem. The platinum-based anti-tumor agent is a DNA-reactive reagent which causes cell cycle arrest at various phases in the cell cycle and induces apoptosis. Hence, the drug active pathway plays an important role in drug resistance in the cellular system. It is also a very important issue in the identification and validation of drug target genes by supplying their interactive relationships. This approach elucidated the particular chemoresistance-associated pathways in large biological interaction networks. Genes deemed relevant for chemotherapy resistance were also likewise determined. After identifying the chemoresistance-associated pathways, the scoring procedure filtered the significant pathways according to the genes' differential expressions. Consequently, this allowed for the identification of dissimilarities between the responses of chemosensitivity to the chemoresistance expression cancer data. In particular, we identified genes and pathway components such as the Hedgehog signalling pathway, the WNT signalling pathway, and the notch signalling pathway, that are relevant to chemoresistance for ovarian and lung cancer. The advantage of comparison analysis is in recognizing the divergent and convergent mechanisms of chemoresistance between cancers. Through systems biology methods, biologists can perform a comprehensive survey to upon which to base hypothetical assumptions.

The advantages of pathway intersections analysis include: revealing whether different cancers have same chemoresistant mechanisms, and determining whether some common genes involved in these chemoresistant mechanisms. As expected, we observed a great deal of correspondence between the response interactions of ovarian and lung cancer expression data by intersecting pathways. The analysis of platinum-based chemotherapeutic agents revealed insights into common responses among the chemoresistant mechanisms as well as the candidate genes such as Bcl-2, AHR and, most importantly, SOD1. The results also indicate that the WNT signaling pathway, the Notch signaling pathway and the FAK pathway are involved in ovarian and lung chemoresistance. Therefore, further analysis of our computational experiment results may reveal additional chemoresistance mechanisms, which indicates this approach can anticipate target identification and chemoresistance in the future development of cancer drugs.

Pathways with a dissimilar response to that of known modes of biological action can be easily identified early in the drug development process to avert repeated and costly clinical trails. This approach reveals chemoresistance-associated pathways in scilicon and enables easier comparisons with the generated graphs. Furthermore, by exploring signature genes involved in chemoresistance mechanisms, this approach sheds light on how these genes or pathways interact with each other, and provides analysis of the betweenness centrality and degree values of genes in pathways. In summary, this method is sufficiently flexible to accommodate various types of biological network information and experimental data, and offers not only insights into the mechanisms of chemoresistance but also provides information on potential candidate target genes for future drug-development efforts.

\section{Additional material}

Additional file 1: Pathway lists. The pathways used in this study are shown in additional file 1.

Additional file 2: Representation of the notations used by this work This additional file demonstrates the notations used in pathway representation.

Additional file 3: Significant results following pathway intersections. The main analysis of this experiment focused on whether different cancers have same chemoresistant mechanisms and whether these chemoresistant mechanisms share some genes in common. We demonstrated the concept and the numeric results in this supplementary file.

Additional file 4: Pathway intersection results and analysis. We demonstrated another pathway intersection result. In this pathway, the start node and end node are NF-KB and CENTG2, respectively. Several sub-pathways were involved in this experimental result, such as Apoptosis, Focal adhesion, and Jak-STAT signal pathway. More detailed analysis was shown in this file.

\section{Acknowledgements}

This research work was supported in part by Research Grant NSC98-2218-E231-002 from the National Science Council, Taiwan. We thank Dr. Lan Chun Tu for the advice and critical reading of the manuscript.

\section{Author details}

'Department of Computer Science and Information Engineering, Ching Yun University, No. 229, Jiansing Road, Jhongli City, Taoyuan County 320, Taiwan. ${ }^{2}$ Department of Computer Science and Information Engineering, National 
Cheng Kung University, No. 1, University Road, Tainan City 701, Taiwan. ${ }^{3}$ Department of Biochemistry, Kaoshiung Medical University, Shih-Chuan 1st Road, Kaohsiung, 807, Taiwan.

\section{Authors' contributions}

SYC carried out the design of study, participated in the system implementation, wrote the program codes and drafted the manuscript. $\mathrm{AMH}$ participated in analysis the experimental results and helped to draft the manuscript. JHC participated in drafting the manuscript and WSC participated in writing the program codes. All authors read and approved the final manuscript.

\section{Competing interests}

The authors declare that they have no competing interests.

Received: 23 August 2010 Accepted: 24 March 2011

Published: 24 March 2011

\section{References}

1. Bosch TM: Pharmacogenomics of drug-metabolizing enzymes and drug transporters in chemotherapy. Methods Mol Biol 2008, 448:63-76.

2. Meisel P: Arylamine $\mathrm{N}$-acetyltransferases and drug response. Pharmacogenomics 2002, 3(3):349-366.

3. Gottesman MM, Fojo T, Bates SE: Multidrug resistance in cancer: role of ATP-dependent transporters. Nat Rev Cancer 2002, 2(1):48-58.

4. Hembruff SL, Laberge ML, Villeneuve DJ, Guo B, Veitch Z, Cecchetto M, Parissenti AM: Role of drug transporters and drug accumulation in the temporal acquisition of drug resistance. BMC Cancer 2008, 8:318.

5. Torigoe $T$, Izumi $H$, Ishiguchi $H$, Yoshida $Y$, Tanabe M, Yoshida T, Igarashi T, Niina I, Wakasugi T, Imaizumi $T$, et al: Cisplatin resistance and transcription factors. Curr Med Chem Anticancer Agents 2005, 5(1):15-27.

6. Liu LZ, Zhou XD, Qian G, Shi X, Fang J, Jiang BH: AKT1 amplification regulates cisplatin resistance in human lung cancer cells through the mammalian target of rapamycin/p70S6K1 pathway. Cancer Res 2007, 67(13):6325-6332.

7. Wu C, Wangpaichitr M, Feun L, Kuo MT, Robles C, Lampidis T, Savaraj N: Overcoming cisplatin resistance by $\mathrm{mTOR}$ inhibitor in lung cancer. $\mathrm{Mol}$ Cancer 2005, 4(1):25

8. Kang HC, Kim IJ, Park JH, Shin Y, Ku JL, Jung MS, Yoo BC, Kim HK, Park JG: Identification of genes with differential expression in acquired drugresistant gastric cancer cells using high-density oligonucleotide microarrays. Clin Cancer Res 2004, 10(1 Pt 1):272-284.

9. Tanaka T, Tanimoto K, Otani K, Satoh K, Ohtaki M, Yoshida K, Toge T, Yahata $\mathrm{H}$, Tanaka S, Chayama K, et al: Concise prediction models of anticancer efficacy of 8 drugs using expression data from 12 selected genes. Int J Cancer 2004, 111(4):617-626.

10. Nakatsu N, Yoshida Y, Yamazaki K, Nakamura T, Dan S, Fukui Y, Yamori T: Chemosensitivity profile of cancer cell lines and identification of genes determining chemosensitivity by an integrated bioinformatical approach using cDNA arrays. Mol Cancer Ther 2005, 4(3):399-412.

11. Cabusora L, Sutton E, Fulmer A, Forst CV: Differential network expression during drug and stress response. Bioinformatics 2005, 21(12):2898-2905.

12. Riedel RF, Porrello A, Pontzer E, Chenette EJ, Hsu DS, Balakumaran B, Potti A, Nevins J, Febbo PG: A genomic approach to identify molecular pathways associated with chemotherapy resistance. Mol Cancer Ther 2008, 7(10):3141-3149.

13. Rabik CA, Dolan ME: Molecular mechanisms of resistance and toxicity associated with platinating agents. Cancer Treat Rev 2007, 33(1):9-23.

14. Kelland $L:$ The resurgence of platinum-based cancer chemotherapy. Nat Rev Cancer 2007, 7(8):573-584.

15. Schaefer CF, Anthony K, Krupa S, Buchoff J, Day M, Hannay T, Buetow KH: PID: the Pathway Interaction Database. Nucleic Acids Res 2009, , 37 Database: D674-679.

16. Matys V, Fricke E, Geffers R, Gossling E, Haubrock M, Hehl R, Hornischer K, Karas D, Kel AE, Kel-Margoulis OV, et al: TRANSFAC: transcriptional regulation, from patterns to profiles. Nucleic Acids Res 2003, 31(1):374-378.

17. Kanehisa M, Araki M, Goto S, Hattori M, Hirakawa M, Itoh M, Katayama T, Kawashima S, Okuda S, Tokimatsu T, et al: KEGG for linking genomes to life and the environment. Nucleic Acids Res 2008, , 36 Database: D480-484.

18. Kanehisa M, Goto S, Hattori M, Aoki-Kinoshita KF, Itoh M, Kawashima S, Katayama T, Araki M, Hirakawa M: From genomics to chemical genomics: new developments in KEGG. Nucleic Acids Res 2006, , 34 Database: D354-357.

19. Peters D, Freund J, Ochs RL: Genome-wide transcriptional analysis of carboplatin response in chemosensitive and chemoresistant ovarian cancer cells. Mol Cancer Ther 2005, 4(10):1605-1616.

20. Almeida GM, Duarte TL, Farmer PB, Steward WP, Jones GD: Multiple endpoint analysis reveals cisplatin damage tolerance to be a chemoresistance mechanism in a NSCLC model: implications for predictive testing. Int J Cancer 2008, 122(8):1810-1819.

21. Barrett T, Troup DB, Wilhite SE, Ledoux P, Rudnev D, Evangelista C, Kim IF, Soboleva A, Tomashevsky M, Marshall KA, et al: NCBI GEO: archive for high-throughput functional genomic data. Nucleic Acids Res 2009, , 37 Database: D885-890.

22. Yen JY: Finding the K Shortest Loopless Paths in a Network. Management Science 1971, 17(11):712-716.

23. Hour TC, Lai YL, Kuan Cl, Chou CK, Wang JM, Tu HY, Hu HT, Lin CS, Wu WJ, Pu YS, et al: Transcriptional up-regulation of SOD1 by CEBPD: a potential target for cisplatin resistant human urothelial carcinoma cells. Biochem Pharmacol 2010, 80(3):325-334.

24. Wood RD, Mitchell M, Lindahl T: Human DNA repair genes, 2005. Mutat Res 2005, 577(1-2):275-283.

25. Kohno K, Uchiumi T, Niina I, Wakasugi T, Igarashi T, Momii Y, Yoshida T, Matsuo K, Miyamoto N, Izumi H: Transcription factors and drug resistance. Eur J Cancer 2005, 41(16):2577-2586

26. Gery S, Tanosaki S, Hofmann WK, Koppel A, Koeffler HP: C/EBPdelta expression in a BCR-ABL-positive cell line induces growth arrest and myeloid differentiation. Oncogene 2005, 24(9):1589-1597.

27. Huang AM, Montagna C, Sharan S, Ni Y, Ried T, Sterneck E: Loss of CCAAT/ enhancer binding protein delta promotes chromosomal instability. Oncogene 2004, 23(8):1549-1557.

28. Sanford DC, DeWille JW: C/EBPdelta is a downstream mediator of IL-6 induced growth inhibition of prostate cancer cells. Prostate 2005, 63(2):143-154.

29. Thangaraju M, Rudelius M, Bierie B, Raffeld M, Sharan S, Hennighausen L, Huang AM, Sterneck E: C/EBPdelta is a crucial regulator of pro-apoptotic gene expression during mammary gland involution. Development 2005, 132(21):4675-4685

30. Ideker T, Ozier O, Schwikowski B, Siegel AF: Discovering regulatory and signalling circuits in molecular interaction networks. Bioinformatics 2002 18(Suppl 1):S233-240.

31. Matthews L, Gopinath G, Gillespie M, Caudy M, Croft D, de Bono B, Garapati P, Hemish J, Hermjakob H, Jassal B, et al: Reactome knowledgebase of human biological pathways and processes. Nucleic Acids Res 2009, , 37 Database: D619-622.

32. Eltabbakh GH, Awtrey CS: Current treatment for ovarian cancer. Expert Opin Pharmacother 2001, 2(1):109-124.

33. Hibi K, Takahashi T, Sekido $Y$, Ueda R, Hida T, Ariyoshi Y, Takagi H: Coexpression of the stem cell factor and the c-kit genes in small-cell lung cancer. Oncogene 1991, 6(12):2291-2296.

34. Krystal GW, Hines SJ, Organ CP: Autocrine growth of small cell lung cancer mediated by coexpression of c-kit and stem cell factor. Cancer Res 1996, 56(2):370-376.

35. Raspollini MR, Amunni G, Villanucci A, Baroni G, Taddei A, Taddei GL: c-KIT expression and correlation with chemotherapy resistance in ovarian carcinoma: an immunocytochemical study. Ann Oncol 2004, 15(4):594-597.

36. Hennessy BT, Smith DL, Ram PT, LU Y, Mills GB: Exploiting the PI3K/AKT pathway for cancer drug discovery. Nat Rev Drug Discov 2005, 4(12):988-1004.

37. Ohta T, Ohmichi M, Hayasaka T, Mabuchi S, Saitoh M, Kawagoe J, Takahashi K, Igarashi H, Du B, Doshida M, et al: Inhibition of phosphatidylinositol 3-kinase increases efficacy of cisplatin in in vivo ovarian cancer models. Endocrinology 2006, 147(4):1761-1769.

38. Lee S, Choi EJ, Jin C, Kim DH: Activation of PI3K/Akt pathway by PTEN reduction and PIK3CA mRNA amplification contributes to cisplatin resistance in an ovarian cancer cell line. Gynecol Oncol 2005, 97(1):26-34

39. L'Esperance S, Popa I, Bachvarova M, Plante M, Patten N, Wu L, Tetu B, Bachvarov D: Gene expression profiling of paired ovarian tumors obtained prior to and following adjuvant chemotherapy: molecular signatures of chemoresistant tumors. Int J Oncol 2006, 29(1):5-24.

40. Hintze A, Adami C: Evolution of complex modular biological networks. PLoS Comput Biol 2008, 4(2):e23. 
41. Siddik ZH: Cisplatin: mode of cytotoxic action and molecular basis of resistance. Oncogene 2003, 22(47):7265-7279.

42. Gagnon V, Mathieu I, Sexton E, Leblanc K, Asselin E: AKT involvement in cisplatin chemoresistance of human uterine cancer cells. Gynecol Oncol 2004, 94(3):785-795.

43. Ali IU, Schriml LM, Dean M: Mutational spectra of PTEN/MMAC1 gene: a tumor suppressor with lipid phosphatase activity. J Natl Cancer Inst 1999, 91(22):1922-1932.

44. Myers MP, Pass I, Batty IH, Van der Kaay J, Stolarov JP, Hemmings BA, Wigler MH, Downes CP, Tonks NK: The lipid phosphatase activity of PTEN is critical for its tumor supressor function. Proc Natl Acad Sci USA 1998, 95(23):13513-13518.

45. Gatcliffe TA, Monk BJ, Planutis K, Holcombe RF: Wnt signaling in ovarian tumorigenesis. Int J Gynecol Cancer 2008, 18(5):954-962.

46. Ricken A, Lochhead P, Kontogiannea M, Farookhi R: Wnt signaling in the ovary: identification and compartmentalized expression of wnt-2, wnt2b, and frizzled-4 mRNAs. Endocrinology 2002, 143(7):2741-2749.

47. Yao HH, Matzuk MM, Jorgez CJ, Menke DB, Page DC, Swain A, Capel B: Follistatin operates downstream of Wnt4 in mammalian ovary organogenesis. Dev Dyn 2004, 230(2):210-215.

48. Wang Z, Li Y, Banerjee S, Sarkar FH: Emerging role of Notch in stem cells and cancer. Cancer Lett 2009, 279(1):8-12.

49. Dobrzycka B, Terlikowski SJ, Kowalczuk O, Niklinska W, Chyczewski L, Kulikowski M: Mutations in the KRAS gene in ovarian tumors. Folia Histochem Cytobiol 2009, 47(2):221-224.

50. Auner V, Kriegshauser G, Tong D, Horvat R, Reinthaller A, Mustea A, Zeillinger R: KRAS mutation analysis in ovarian samples using a high sensitivity biochip assay. BMC Cancer 2009, 9:111.

\section{Pre-publication history}

The pre-publication history for this paper can be accessed here:

http://www.biomedcentral.com/1755-8794/4/23/prepub

doi:10.1186/1755-8794-4-23

Cite this article as: Chao et al: An integrative approach to identifying cancer chemoresistance-associated pathways. BMC Medical Genomics 2011 4:23.

\section{Submit your next manuscript to BioMed Central and take full advantage of:}

- Convenient online submission

- Thorough peer review

- No space constraints or color figure charges

- Immediate publication on acceptance

- Inclusion in PubMed, CAS, Scopus and Google Scholar

- Research which is freely available for redistribution

Submit your manuscript at www.biomedcentral.com/submit 